\title{
JNK pathway activation is able to synchronize neuronal death and glial phagocytosis in Drosophila
}

\author{
J Shklover ${ }^{1}$, K Mishnaevski $^{1}$, F Levy-Adam ${ }^{1}$ and E Kurant ${ }^{* 1}$
}

Glial phagocytosis of superfluous neurons and damaged or aberrant neuronal material is crucial for normal development and maintenance of the CNS. However, the molecular mechanisms underlying the relationship between neuronal death and glial phagocytosis are poorly understood. We describe a novel mechanism that is able to synchronize neuronal cell death and glial phagocytosis of dying neurons in the Drosophila embryonic CNS. This mechanism involves c-Jun N-terminal kinase (JNK) signaling, which is required for developmental apoptosis of specific neurons during embryogenesis. We demonstrate that the dJNK pathway gain-of-function in neurons leads to dJNK signaling in glia, which results in upregulation of glial phagocytosis. Importantly, this promotion of phagocytosis is not mediated by upregulation of the glial phagocytic receptors SIMU and DRPR, but by increasing glial capacity to degrade apoptotic particles inside phagosomes. The proposed mechanism may be important for removal of damaged neurons in the developing and mature CNS.

Cell Death and Disease (2015) 6, e1649; doi:10.1038/cddis.2015.27; published online 19 February 2015

During normal development of the central nervous system (CNS) a large number of neurons die through apoptosis ${ }^{1-4}$ and are efficiently removed by phagocytic glia., ${ }^{2,5}$ In the developing and adult CNS, different types of damage may occur, leading to neuronal injury. In these situations glia become reactive, upregulate their phagocytic ability and remove neuronal debris from the CNS. ${ }^{6-9}$ However, the molecular mechanisms underlying the marked changes in glia remain elusive.

Drosophila glia are highly homologous functionally and molecularly to their mammalian counterparts. ${ }^{10-12}$ During embryogenesis, glial phagocytosis is determined by developmentally regulated expression of the phagocytic receptors Six Microns Under (SIMU) and Draper (DRPR), which does not depend on apoptosis. ${ }^{13-15}$ However, it is not clear how embryonic glia perform when neural damage occurs.

Recently, it has been shown that adult Drosophila glia become reactive in response to axotomy through Drosophila C-Jun N-terminal kinase (dJNK)-mediated activation of the phagocytic receptor DRPR. ${ }^{9}$ The Drosophila genome contains only a single JNK homolog, basket (bsk), as compared with jnk1, jnk2 and jnk3 genes in mammals. ${ }^{16,17}$ As in mammals, activation of the JNK pathway in Drosophila initiates target gene transcription through phosphorylationregulated activation of the AP-1s dJUN/JRA and dFOS/KAY. BSK's upstream JNK kinases are dMkk4 and Hemipterous (HEP) (Figure 1h).

dJNK signaling is essential for morphogenetic processes, wound healing, response to pathogens, tumor development, compensatory cell proliferation and apoptosis. ${ }^{18}$ In mammals, jnk1/jnk2-double knockout mice exhibit abnormal development of the embryonic brain, likely through inhibition of apoptosis, ${ }^{19}$ but there is little data about the role of the JNK pathway during Drosophila CNS development. ${ }^{20}$

Here, we show that the dJNK pathway is able to connect between neuronal death and upregulation of glial phagocytosis during Drosophila embryogenesis. Although the physiologically relevant role of this phenomenon is not yet clear, our model provides a potential mechanistic basis for connecting these two processes. We demonstrate that the dJNK pathway is normally involved in developmental neuronal apoptosis, but not in glial phagocytosis of apoptotic neurons. However, gain-of-function of dJNK signaling in embryonic neurons induces dJNK pathway activation in glia, which promotes glial phagocytosis of apoptotic cells. Importantly, this upregulation of phagocytosis is not accompanied by increased expression levels of the glial phagocytic receptors SIMU and DRPR or elevated engulfment ability of phagocytic glia, but it promotes degradation of engulfed apoptotic particles. Our work recognizes dJNK signaling as a possible mechanism of upregulation of glial phagocytosis through enhancement of glial capacity to degrade apoptotic particles.

\section{Results}

The dJNK pathway is active in the embryonic CNS in specific neurons and is involved in developmental neuronal apoptosis. Massive neuronal apoptosis takes

\footnotetext{
${ }^{1}$ Department of Genetics and Developmental Biology, Faculty of Medicine, The Rappaport Family Institute for Research in the Medical Sciences, Technion—-Israel Institute of Technology, Haifa 31096, Israel

${ }^{*}$ Corresponding author: E Kurant, Department of Genetics and Developmental Biology, Faculty of Medicine, The Rappaport Family Institute for Research in the Medical Sciences, Technion-Israel Institute of Technology, Efron Street, PO Box 9697, Haifa 31096, Israel. Tel: +972 48295383 ; Fax: +972 48295403 ; E-mail: kurante@tx.technion.ac.il

Abbreviations: AP-1, activator protein 1; BSK, Basket; CA, constitutively active; CNS, central nervous system; DAC, Dachshund; dJNK, Drosophila c-Jun N-terminal kinase; DN, dominant negative; DRPR, Draper; EVE, even-skipped; HEP, Hemipterous; HID, Head involution detective; JNK, c-Jun N-terminal kinase; LT, LysoTracker; nGFP, nuclear green fluorescent protein; Puc, Puckered; REPO, Reverse Polarity; SIMU, Six Microns Under; wt, wild type

Received 04.8.14; revised 12.1.15; accepted 13.1.15; Edited by A Yaron
} 
place in the Drosophila CNS during late stages of embryogenesis. ${ }^{13,21}$ As the dJNK pathway has been shown to induce ectopic apoptosis in imaginal epithelia of eye and wing disks ${ }^{22,23}$ and bsk RNA is specifically expressed in the late embryonic CNS, ${ }^{16}$ we examined whether the dJNK pathway is active in the embryonic CNS. We followed expression of a lac $Z$ enhancer trap in the gene puckered (puclac $Z$ reporter), which is a target of the dJNK pathway and
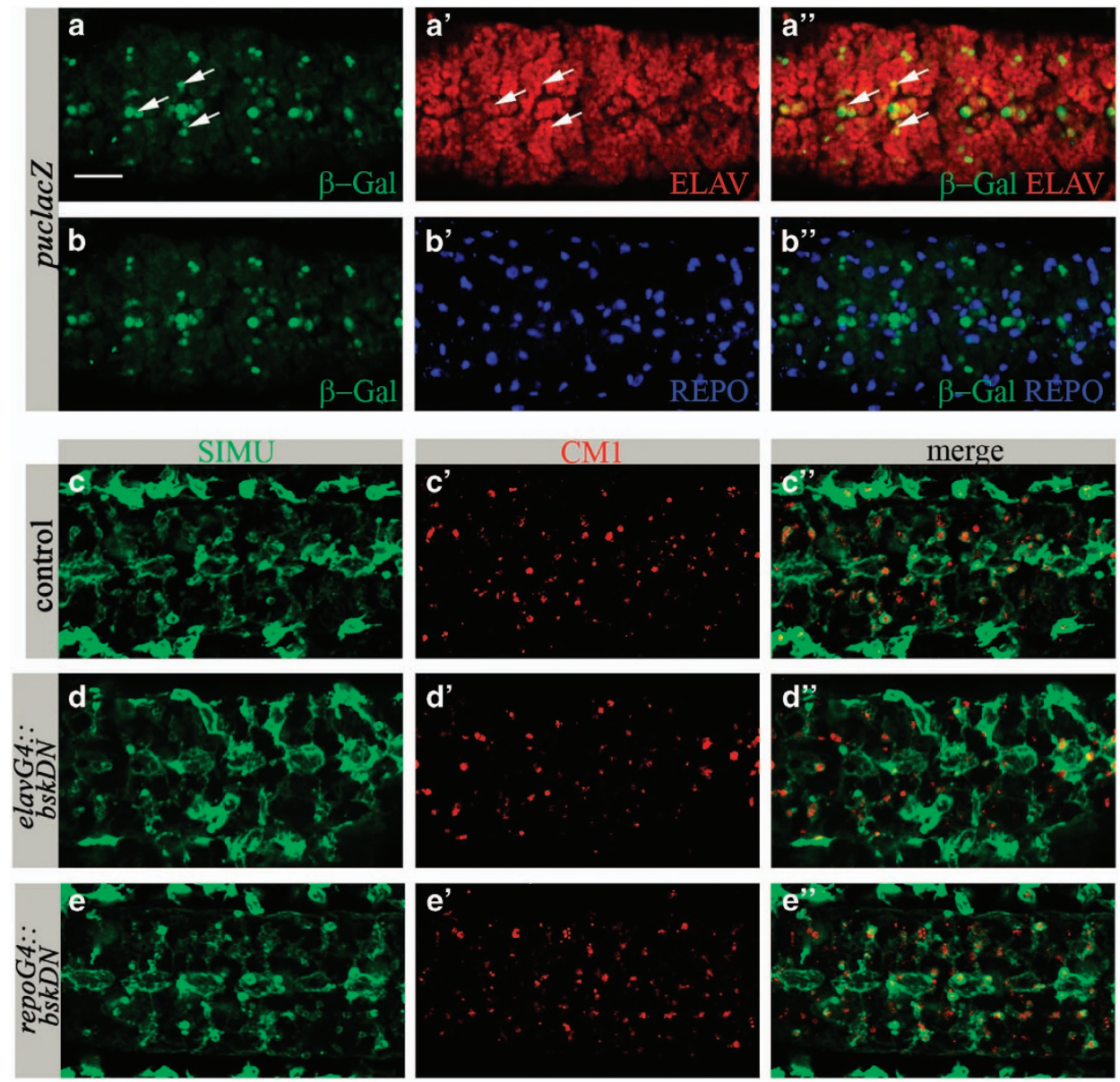

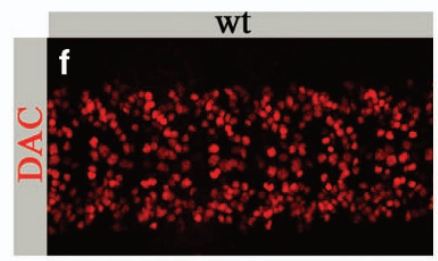

g Volume of apoptotic particles (\%)

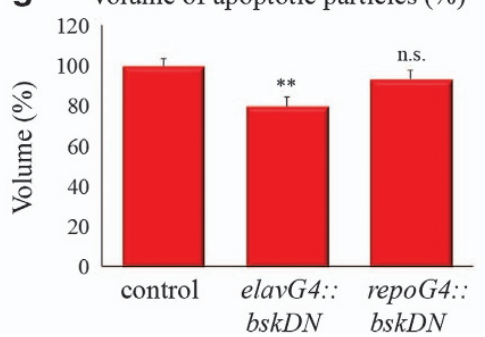

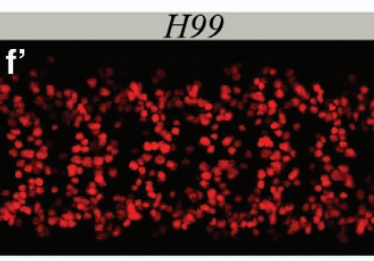

h

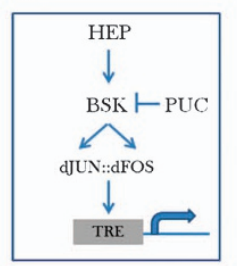

i

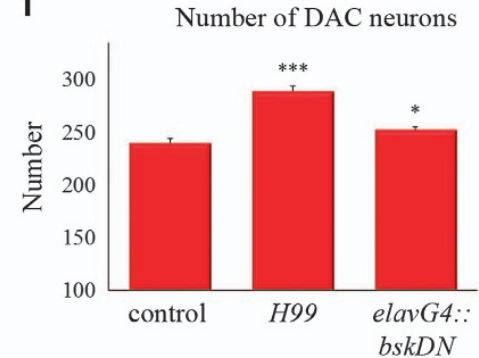

Figure 1 The dJNK pathway is active in embryonic neurons and is required for neuronal apoptosis. (a- $\mathbf{f}^{\prime \prime}$ ) Projections from confocal stacks of the embryonic CNS at stage 16; ventral view. Bar $20 \mu \mathrm{m}$. (a-b") Embryos expressing puclacZ reporter stained with anti- $\beta$-Gal (green), anti-ELAV (red) and anti-REPO (blue) antibodies. Some neurons exhibiting puclacZ expression are marked with arrows. (c- $\left.\mathbf{e}^{\prime \prime}\right)$ Control embryos and embryos expressing bskDN in neurons (elavGal4::bskDNK53R) or glia (repoGal4::bskDNK53R) are labeled with anti-activated caspase 3 (CM1, red) and glia (anti-SIMU, green). (f- $\mathbf{f}^{\prime \prime}$ ) Neurons labeled with anti-DAC (red) in control, H99, and elavGal4::bskDNK53R embryos. ( $\mathbf{g}$ and i) Columns represent mean total volume of apoptotic particles (g) or DAC-positive neurons (i) within confocal stacks of the CNS, \pm S.E.M., $n=7-12$; asterisks indicate statistical significance versus control, as determined by the Student's $t$-test, ${ }^{* \star} P<0.0001,{ }^{\star \star} P<0.002,{ }^{*} P<0.04, N S$ (not significant) $P>0.05$. (h) Schematic representation of dJNK signaling to the nucleus to induce gene expression 

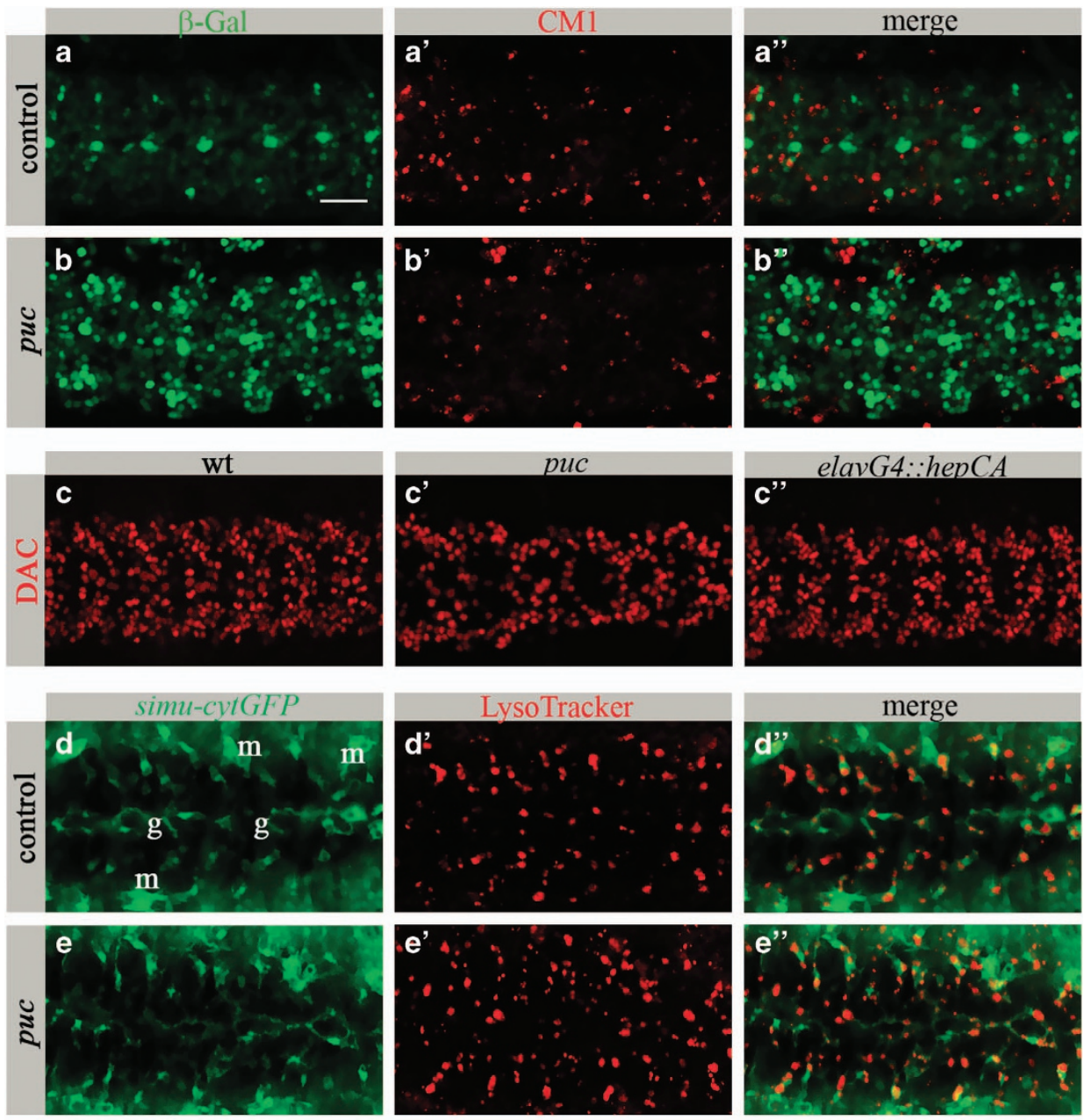

f Volume of

g

apoptotic particles (\%)

Number of DAC neurons
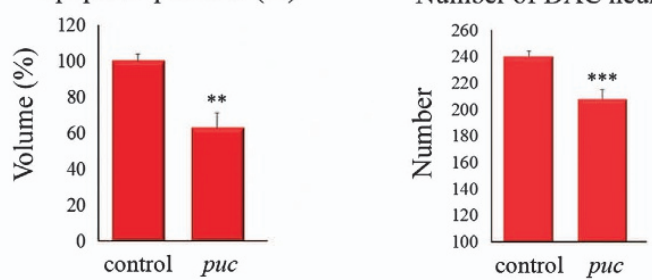

h

Number of phagosomes

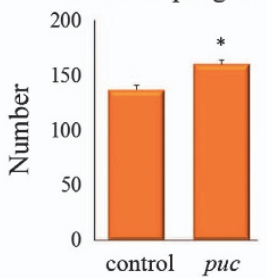

Figure 2 Gain-of-function of the dJNK pathway reduces the volume of apoptotic particles in the CNS. (a-c) Projections from confocal stacks of the embryonic CNS at stage 16; ventral view. Bar $20 \mu \mathrm{m}$. (a-b") Embryos expressing puclacZ reporter stained with anti- $\beta$-Gal (green) and CM1 (red) antibodies. (a-a") puclacZ heterozygous embryos as a control. (b-b") puclacZ homozygous mutant embryos. (c-c") Neurons labeled with anti-DAC (red). (d-e $\mathbf{e}^{\prime \prime}$ ) Projections from confocal stacks of the embryonic CNS of stage-16 live embryos; ventral view. simu-cytGFP reporter marks glia (g) and macrophages $(\mathrm{m})$ in green. Phagosomes are marked with LysoTracker (red). (f) Volume of apoptotic particles within CNS sections. Columns represent mean total volume of apoptotic particles within confocal stacks of the CNS, \pm S.E.M., $n=7-8$; asterisks indicate statistical significance versus control, as determined by the Student's t-test, ${ }^{* \star} P<0.003$. (g) Quantification of DAC-positive neurons. To count DAC-positive neurons, confocal stacks of the whole CNS (15 sections; total $22.5 \mu \mathrm{m}$ ) were acquired. Columns represent mean total number of DAC-positive neurons in three segments within confocal stacks of the CNS, \pm S.E.M., $n=8-15$; asterisks indicate statistical significance versus control, as determined by the Student's $t$-test, ${ }^{* \star *} P<0.0001$. (h) Number of phagosomes within CNS sections. Columns represent mean total number of phagosomes within confocal stacks of the CNS, \pm S.E.M., $n=6-7$; asterisks indicate statistical significance versus control, as determined by the Student's $t$-test, ${ }^{*} P<0.04$

also inhibits it by a feedback loop. ${ }^{24}$ To examine the embryonic CNS of puclacZ transgenic embryos, we used differential labeling of neurons and glia by specific antibodies against ELAV (Figure 1a) and REPO (Reverse Polarity; Figure 1b), respectively. In these embryos, we detected colocalization of $\beta$-Gal (Figures 1a and b) with ELAV (Figure 1a"), but not with
REPO (Figure 1 $b^{\prime \prime}$ ) staining, suggesting that during late embryogenesis the dJNK pathway is active in specific neurons but not in glia.

To test the role of dJNK signaling in neuronal apoptosis, we knocked down BSK function by expressing its dominantnegative (DN) form, bskDN, specifically in neurons using the 
elavGal4 driver. To evaluate the levels of apoptosis in the CNS, we used an anti-activated caspase 3 antibody (CM1) for labeling apoptotic particles (Figures $1 c^{\prime}, c^{\prime \prime}, d^{\prime}$ and $d^{\prime \prime}$ ). Specific impairment of BSK function in post-mitotic neurons led to a small but significant reduction in apoptotic cell volume as compared with control embryos (Figure 1g), suggesting that inhibition of dJNK signaling prevented caspase activation and apoptosis of certain neurons. To corroborate this result, we assessed neuronal death by quantifying neurons in mutant and control embryos. As using a pan-neuronal marker antiELAV (Figure 1a') is not feasible for counting, we used antiDachshund (DAC), which specifically labels a subset of neurons, whose number is easier to count (Figures $1 \mathrm{f}$ and i). We first verified that DAC-positive neurons die during normal embryogenesis, using the anti-DAC staining of $\mathrm{H} 99$ embryos (Figure 1f). H99 embryos are hid, reaper and grim deficient and lack caspase activation. ${ }^{13,25}$ Counting the DAC-positive neurons in $\mathrm{H} 99$ mutant embryos indicated a significant increase in their number compared with wild-type (wt) embryos (Figure 1i), indicating that these neurons normally die through apoptosis. Importantly, expressing bskDN in glia (repoGal4 driver), did not affect the volume of apoptotic particles and the shape of glial cells (Figures 1e- $\mathrm{e}^{\prime \prime}$ and g) as compared with control embryos (Figures $1 \mathrm{c}-\mathrm{c}^{\prime \prime}$ and g). These results indicate that the dJNK pathway does not function in embryonic glia, which is consistent with the lack of puclacZ reporter expression in glial cells (Figure $\left.1 b^{\prime \prime}\right)$.

Gain-of-function of dJNK signaling leads to upregulation of glial phagocytosis. Given that dJNK signaling is involved in apoptosis of certain neurons, we expected its gain-offunction would lead to higher apoptosis rates in the CNS. Therefore, we tested levels of apoptosis in $P\{A 92\}$ puc ${ }^{E 69}$ (puc) mutant embryos, in which the dJNK pathway is overactivated because of the removal of Puc inhibition. puc mutant embryos exhibited a large volume of apoptotic particles outside the CNS (Supplementary Figure S1), resulting probably from the excessive dJNK pathway activity at earlier embryogenesis. Surprisingly, a significantly lower volume of apoptotic particles was detected inside the CNS of these embryos compared with control heterozygous embryos (Figures 2a', a", b', b" and f).

A reduction in the volume of apoptotic particles would be detected when less apoptosis occurs or alternatively, when phagocytosis of apoptotic particles is more efficient, leading to their faster degradation inside phagocytes and therefore to their lower amount. To assess neuronal loss, we quantified DAC-positive neurons in puc mutant and control embryos and detected a significantly lower number in the mutants (Figures $2 \mathrm{c}, \mathrm{c}^{\prime}$ and g). To test whether the reduced number of DACpositive neurons is a result of apoptosis or of their diminished production, we counted them in mutant embryos at early stage 14 , before a pick of neuronal developmental apoptosis occurs and after the beginning of puc expression in the CNS. ${ }^{21}$ The same number of DAC-positive neurons was detected in mutant and control embryos (Supplementary Figure S2), indicating no defects in their production. Moreover, to test a possible change in cell fate of these neurons in the mutant, we quantified CUT- and EVE-positive neurons, as well as REPO-positive glial cells in stage-16 embryos. None of these cell types showed any increase in their number compared with wt (Supplementary Figure S3; Figure 4e), suggesting DACpositive neurons in puc mutant embryos are rather eliminated through cell death. Therefore, the decreased volume of apoptotic particles observed in mutant embryos indicates that neurons may disappear through caspase-independent cell death or accelerated glial phagocytosis.

To evaluate the level of glial phagocytosis in puc mutants, we used LysoTracker (LT) labeling of phagosomal activity in glial cells (Figures $2 \mathrm{~d}-\mathrm{e}^{\prime \prime}$ ). Wt embryonic glia are highly phagocytic during late embryogenesis, as reflected by LT-positive phagosomes inside GFP-labeled glial cells (Figure $2 d^{\prime \prime}$ ). Quantification of the data revealed significantly higher number of phagosomes in the puc mutant than in the control CNS (Figures 2d', d", e', e $e^{\prime \prime}$ and h), supporting the assumption of upregulated phagocytic activity. These data imply that in puc embryos, the higher level of neuronal death is accompanied by upregulated glial phagocytosis.

Overactivation of dJNK signaling in neurons leads to dJNK signaling in glia. To further understand the molecular basis of increased glial phagocytosis in puc mutants, we introduced a reporter for dJNK activity, TREeGFP that contains Drosophila AP-1-binding sites fused to the eGFP gene ${ }^{9,26}$ (Figure 1h). We observed a robust neuronal expression of TRE-eGFP in puc mutant embryos (Figures $3 b$ and $b^{\prime \prime}$ ); however, not all of these neurons died as suggested by the quantification of DAC-positive neurons (Figure $2 \mathrm{~g}$ ). These data propose that dJNK gain-of-function alone is not sufficient to kill embryonic neurons.

Interestingly, in addition to its neuronal expression, TREeGFP was detected in some glial cells in puc embryos (Figures $3 b-b^{\prime \prime}$ ), indicating that dJNK signaling was activated in glia as well. We confirmed dJNK pathway activation in glia by immunostaining of puc mutants with an anti-dJUN antibody. A significantly higher signal of dJUN was detected in mutant neurons and glia as compared with control embryos (Figures 3c, $c^{\prime \prime}$, d and $d^{\prime \prime}$ ), again demonstrating dJNK pathway activation also in glia.

To test a possible connection between dJNK signaling in neurons and glia as suggested in puc mutants, we sought to test whether specific overactivation of the dJNK pathway in neurons would lead to dJNK signaling in glia. To activate the pathway in neurons, we expressed the constitutively active HEP (hepCA) with the elavGal4 driver. It has been previously shown that elavGal4 is transiently expressed in embryonic glia. ${ }^{27}$ In order to prevent dJNK pathway activation directly in glia, we used tubGal80 repression of the elavGal4 driver until embryonic stage 14, when elavGal4 expression in glia is terminated, ${ }^{27}$ and tested its expression with nuclear GFP. No GFP expression in glia was detected in these embryos, indicating that elavGal4 was solely expressed in neurons (Figures $3 \mathrm{e}-\mathrm{e}^{\prime \prime}$ ). Using the same time courses we expressed hepCA in neurons and followed TRE-eGFP expression. A strong GFP expression was detected in glial cells of these embryos (Figures $\left.3 f-f^{\prime \prime}\right)$, suggesting dJNK signaling in glia as the result of dJNK pathway overactivation specifically in neurons. In these embryos, a significantly lower number of DAC-positive neurons was detected (Figure $3 \mathrm{~g}$ ), but no increase in the volume of apoptotic particles (Figure $3 \mathrm{~h}$ ). 

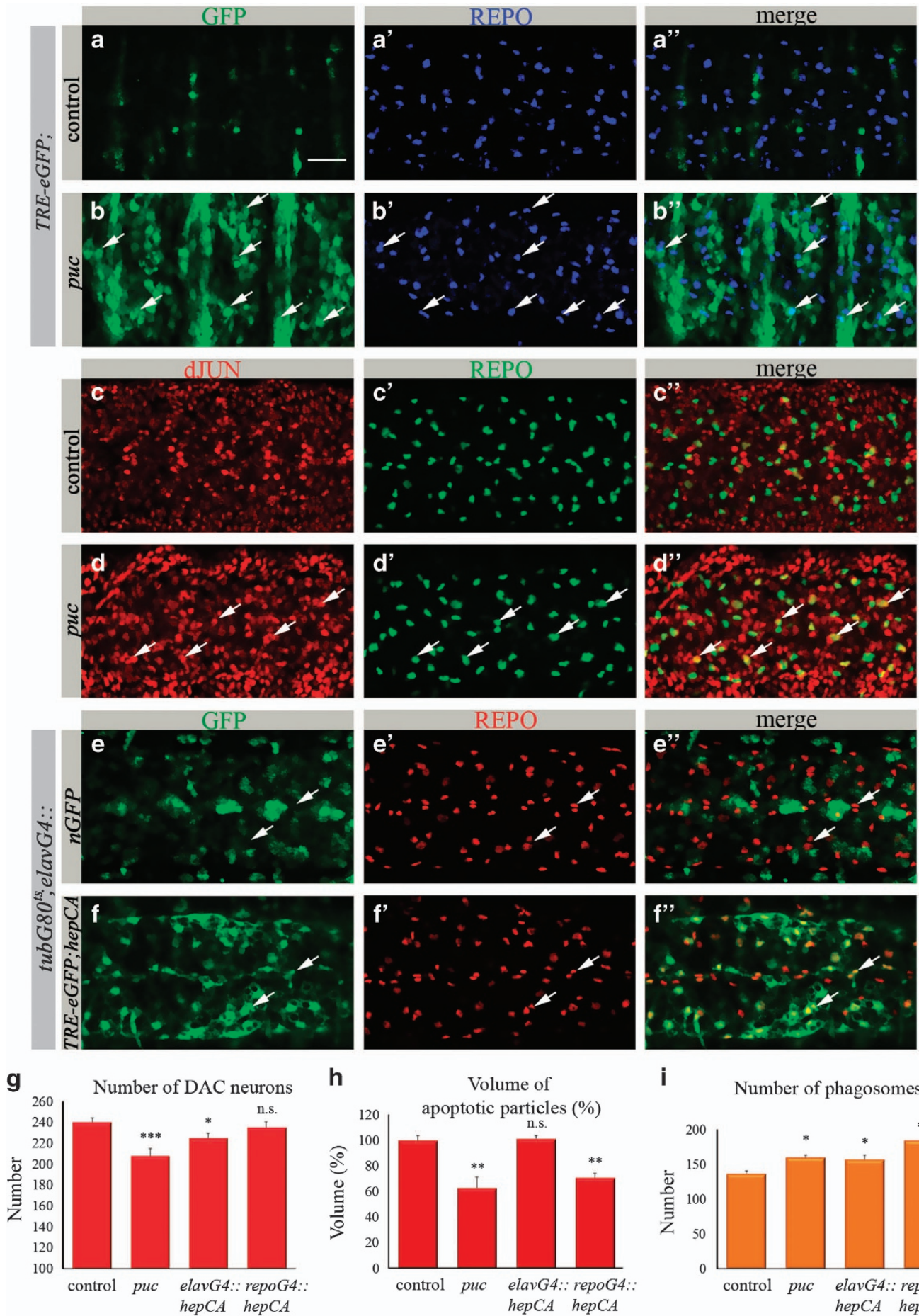

h

Volume of
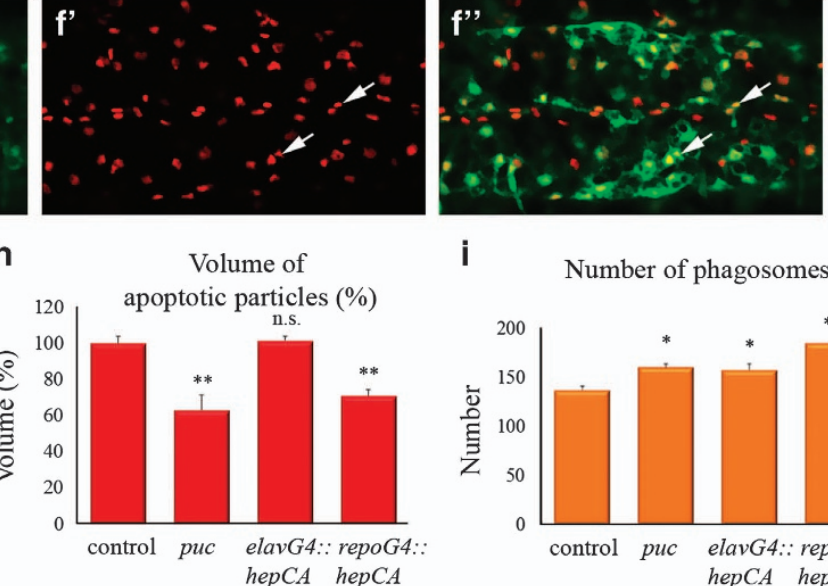

i

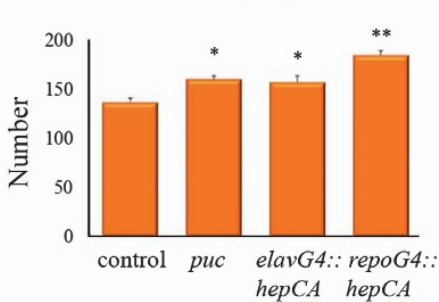

Figure 3 Gain-of-function of dJNK signaling in neurons upregulates glial phagocytosis. (a-f") Projections from confocal stacks of the embryonic CNS at stage 16; ventral view. Bar $20 \mu \mathrm{m}$. (a-b") TRE-eGFP reporter (in green) depicts dJNK pathway activation. REPO-positive glial nuclei are marked in blue. (a-a") repoGal4 control. (b-b") puc E69 mutant embryo. Arrows depict glial cells expressing TRE-eGFP reporter. (c-d") anti-dJUN staining in red and anti-REPO in green. (c-c') repoGal4 control. (d-d") puc E69 mutant embryo. Arrows mark glial cells expressing dJUN. (e-f") Glia in red (anti-REPO). (e-e $\mathbf{e}^{\prime \prime}$ ) tubGal80 ${ }^{\text {ts }}$; elavGal4::nucGFP embryos. No nuclear GFP expression in glia (arrows). $\left(\mathbf{f}-f^{\prime \prime}\right)$ tubGal80 ts; elavGal4::TRE-eGFP;hepCA embryos showing TRE-eGFP expression in glial cells (arrows). (g) Quantification of DAC-positive neurons. Columns represent mean total number of DAC-positive neurons within confocal stacks of the CNS, \pm S.E.M., $n=6-12$. (h) Volume of apoptotiPIc particles within CNS sections. Columns represent mean total volume of apoptotic particles within confocal stacks of the CNS, \pm S.E.M., $n=7-13$. (i) Number of phagosomes within CNS sections. Columns represent mean total number of phagosomes within confocal stacks of the CNS, \pm S.E.M., $n=6-7$. (g-i) Asterisks indicate statistical significance versus control, as determined by the Student's $t$-test, ${ }^{* * *} P<0.0001,{ }^{* *} P<0.003,{ }^{*} P<0.04$, NS (not significant) $P>0.05$ 
Importantly, a significantly elevated number of LT-positive phagosomes (Figure 3i) indicated upregulated phagocytic activity of glial cells. These data suggest that overactivation of the dJNK pathway in neurons leads to dJNK signaling in glia, resulting in promotion of glial phagocytosis.

Glial phagocytosis is upregulated via activation of dJNK signaling in glial cells. To gain deeper insight into dJNK signaling in glia, we examined embryos ectopically expressing the constitutively active HEP specifically in glia (repoGal4::hepCA). In these embryos high levels of TRE-eGFP were detected exclusively in glia (Figures $4 \mathrm{~b}-\mathrm{b}^{\prime \prime}$ ). dJNK pathway activation was also demonstrated by much stronger dJUN in glia as compared with control embryos (Figures $\left.4 c-d^{\prime \prime}\right)$. Strikingly, there was a significantly lower amount of apoptotic particles in the CNS of these embryos as compared with the control (Figure $3 \mathrm{~h}$ ). However, quantification of DAC-labeled neurons showed no significant difference between embryos containing ectopic dJNK signaling in glia and control embryos (Figure $3 \mathrm{~g}$ ), indicating that dJNK pathway activation in glia does not affect neuronal death but rather suggests faster elimination of already dying neurons. This conclusion was confirmed by a significantly higher number of phagosomes in the embryonic glia where the dJNK pathway was activated, than in the control CNS (Figure 3i), demonstrating that dJNK signaling promotes glial phagocytic activity. No significant difference in the amount of REPO-positive nuclei was perceived between mutant and control embryos (Figures $4 a^{\prime}, b^{\prime}, c^{\prime}, d^{\prime}$ and e), indicating that dJNK signaling in glia does not affect glial number.

dJNK signaling promotes glial phagocytosis downstream of SIMU and DRPR. To better understand the molecular mechanisms underlying dJNK-mediated upregulation of glial phagocytosis, we first examined the protein levels of SIMU and DRPR receptors in glia exhibiting activated dJNK signaling. Similar levels and distribution of SIMU and DRPR were observed on glial membranes of control and mutant embryos (Figures 5a, a', b and b). These data are in agreement with our previous results, where we demonstrated that embryonic glia managed to engulf the increased number of apoptotic particles without changing the expression levels of SIMU and DRPR. ${ }^{15}$

To examine whether engulfment and/or degradation of apoptotic particles is enhanced by activation of the dJNK pathway irrespective of simu and drpractivation, we evaluated the ability of glial dJNK signaling to rescue simu and $d r p r$ mutant phenotypes. In the embryonic CNS, simu is required for recognition and engulfment of apoptotic neurons by glia, whereas drpris mostly involved in their degradation inside glial cells. ${ }^{13}$ We activated dJNK signaling specifically in glia of simu (simu;repoGal4::hepCA) or drpr (repoGal4::hepCA;drpr) mutant embryos. simu mutant embryos exhibit a significantly higher volume of apoptotic particles in the $\mathrm{CNS}^{13}$ (Figures 6b', $b^{\prime \prime}$ and f) compared with control embryos (Figures $6 a^{\prime}, a^{\prime \prime}$ and f), and most of them are found outside glial cells labeled by simucytGFP reporter (Figure $\left.6 b^{\prime \prime}\right) .{ }^{13}$ dJNK pathway activation specifically in glia of simu mutant embryos (Supplementary Figure S4) did not rescue the abnormal phagocytosis phenotype (Figures $6 c^{\prime}, c^{\prime \prime}$ and f), suggesting that dJNK signaling is unable to overcome defects in recognition and engulfment of apoptotic particles.

However, we detected a substantial rescue of the drpr mutant phenotype (Figures $6 \mathrm{~d}-\mathrm{d}^{\prime \prime}$ ) by dJNK pathway activation specifically in glia, exhibiting many fewer apoptotic particles inside glial cells, whereas macrophages present outside the CNS display the strong drpr phenotype (Figures 6e-e"). These data place the dJNK pathway activity downstream of SIMU and DRPR function in glial phagocytosis, likely in degradation of apoptotic particles inside glial cells (Figure 7f).

dJNK signaling in glia rescues defects in degradation of apoptotic particles. To further assess how dJNK signaling in glia promotes phagocytosis, we tested components of the basic intracellular phagocytic machinery, which act downstream of simu and drpr in degradation of apoptotic particles. We tested rab5 and the Drosophila homolog of Dynamin, shibire, both required for endosome-phagosome tethering and fusion. ${ }^{28-34}$ Specific expression of DN forms of shibire and rab5 in glia (repoGal4::shibireDN and repoGal4:: rab5DN) caused a strong phagocytosis phenotype, as evaluated by CM1 and anti-SIMU staining (Figures $7 \mathrm{a}^{\prime}$, $a^{\prime \prime}, c^{\prime}, c^{\prime \prime}$ and e). These embryos exhibited large numbers of apoptotic particles in the CNS and, importantly, most of the particles were found inside glial cells, indicating their abnormal or delayed degradation. Strikingly, when we expressed the DN form of shibire together with hepCA specifically in glia (repoGal4::shibireDN; hepCA), we detected a significantly lower volume of apoptotic particles as compared with the shibire mutant alone, indicating a substantial rescue of the abnormal degradation phenotype (Figures 7b', b" and e). Similarly, when hepCA was expressed with the rab5DN form, a significant rescue of the mutant phenotype was observed as well with many fewer apoptotic corpses inside glia (Figures $7 d^{\prime}, d^{\prime \prime}$ and e). These results suggest that dJNK signaling accelerates phagocytosis by stimulating more efficient degradation of engulfed particles (Figure 7f).

\section{Discussion}

Glial phagocytosis has a critical role during Drosophila normal embryonic development, neuronal pruning and axonal degeneration. $8,9,13,15,35-39$ However, much less is known about the molecular mechanisms controlling phagocytic glia when damage occurs in the developing CNS. JNK signaling is one of the major stress response pathways, which often triggers apoptosis. ${ }^{18,40-43}$ The current work shows that dJNK signaling, which is active in certain embryonic neurons, normally dying through apoptosis, is able to synchronize excess neuronal death with upregulation of glial phagocytosis of dying neurons. This promotion of glial phagocytic ability does not affect levels of the phagocytic receptors SIMU and DRPR, but apparently upregulates the intracellular phagocytic machinery responsible for phagosome maturation and degradation of apoptotic particles (Figure 7f). We suggest that this ability of dJNK signaling to promote phagocytic removal of dying cells might be employed for efficient clearance of 

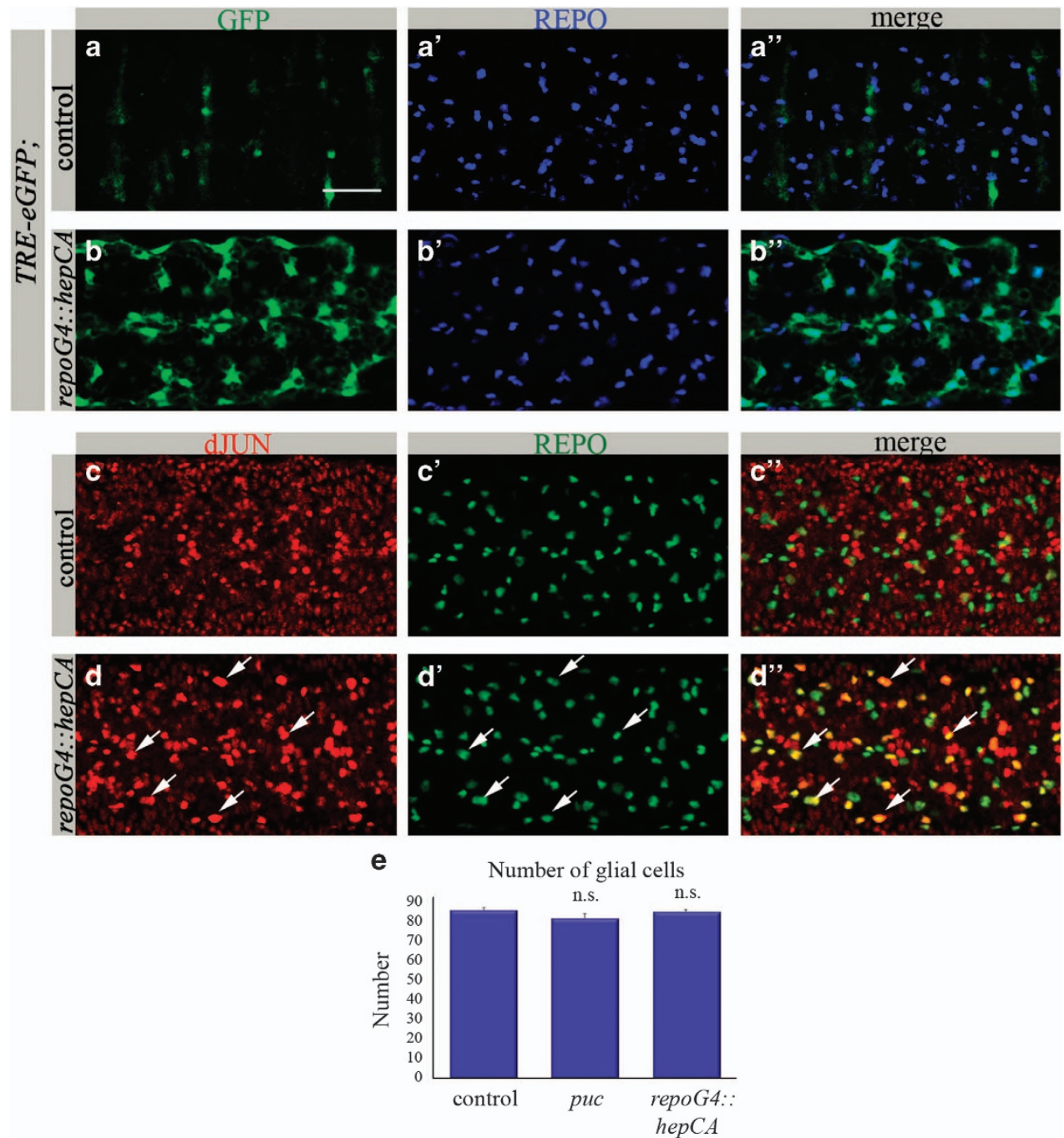

Figure 4 Activation of dJNK signaling in glial cells promotes glial phagocytosis. (a-d") Projections from confocal stacks of the embryonic CNS at stage 16; ventral view. Bar $20 \mu \mathrm{m}$. (a-b") TRE-eGFP reporter (in green) depicts JNK pathway activation. REPO-positive glial nuclei are marked in blue. (a-a") repoGal4 control. (b-b") repoGal4::hepCA mutant embryo. (c- $\left.\mathbf{d}^{\prime \prime}\right)$ anti-dJUN staining in red and anti-REPO in green. (c- $\left.\mathbf{c}^{\prime \prime}\right)$ repoGal4 control. (d- $\mathbf{d}^{\prime \prime}$ ) repoGal4:.:hepCA mutant embryo. Arrows mark glial cells expressing dJUN. (e) Number of REPO-positive nuclei within CNS sections. Columns represent mean total number of glial nuclei within confocal stacks of the CNS, \pm S.E.M., $n=5-8 ;$ NS (not significant) $P>0.05$, as determined by the Student's $t$-test. There is no significant difference in number of glial nuclei between repoGal4::hepCA, puc ${ }^{E 69}$ mutant and control embryos
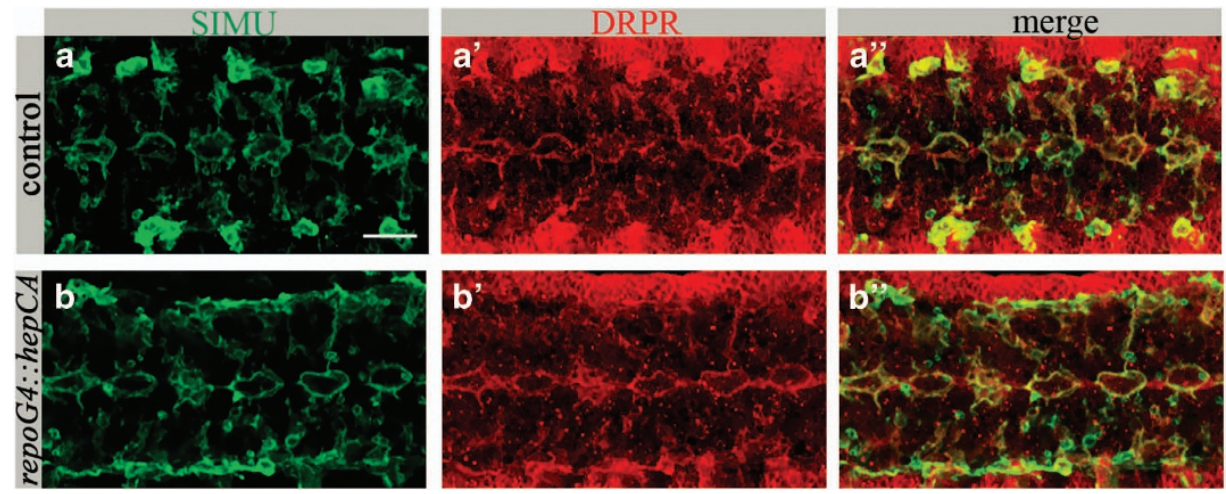

Figure 5 dJNK activation in glia does not affect SIMU or DRPR expression. (a-b") Projections from confocal stacks of the CNS at embryonic stage 16, ventral view. Bar $20 \mu \mathrm{m}$. (a-a") repoGal4 control. (b-b") repoGal4::hepCA embryo showing no change in anti-SIMU (green) or anti-DRPR (red) staining 

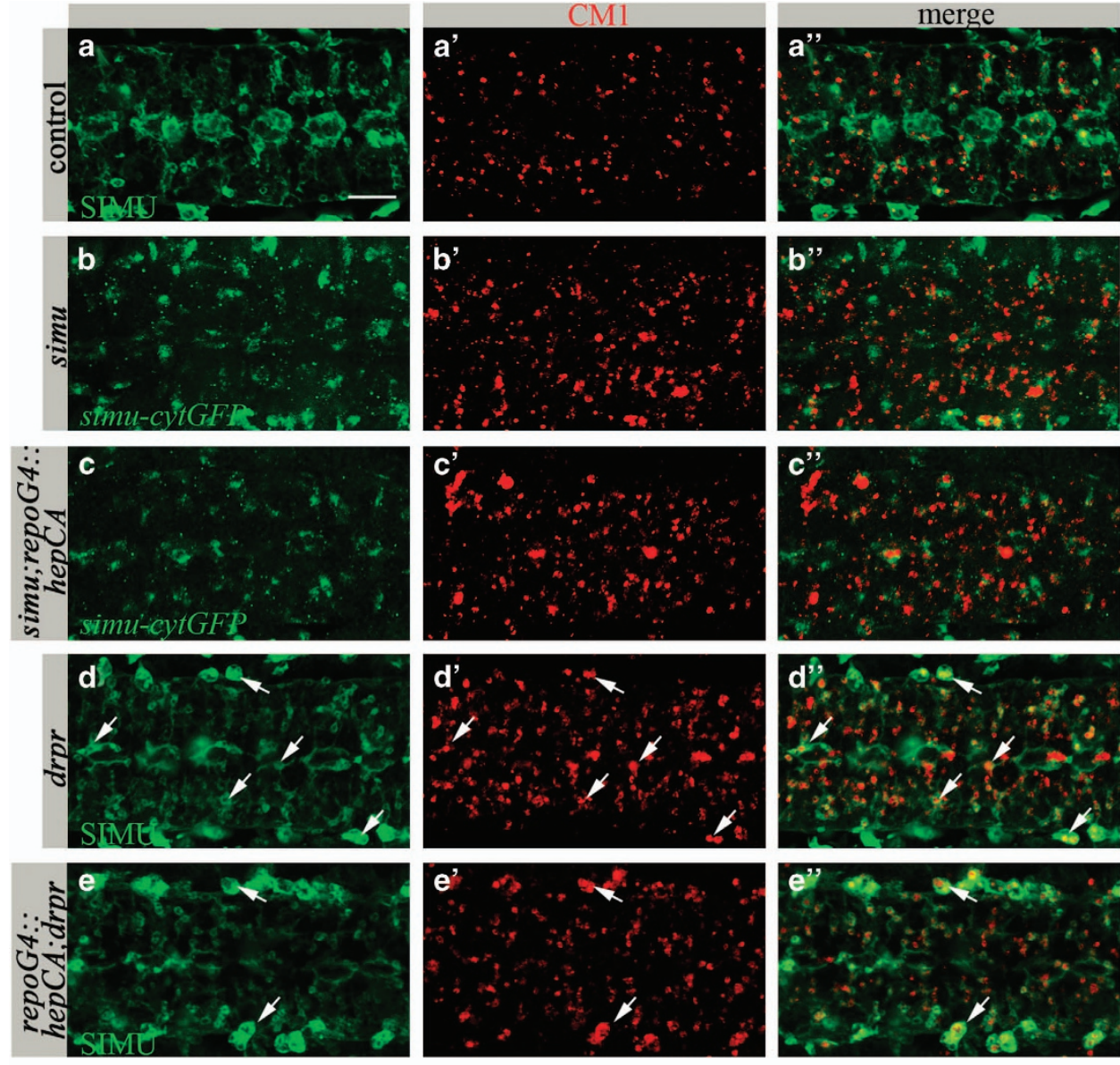

f

Volume of apoptotic particles (\%)

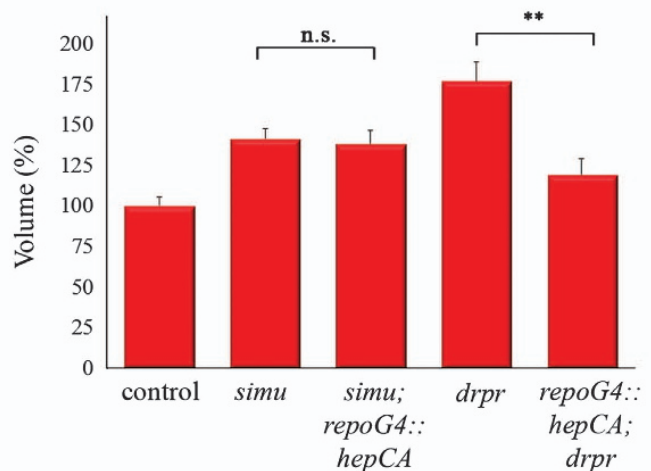

Figure 6 dJNK signaling in glia does not rescue the simu phenotype, whereas it substantially rescues the drpr phenotype. (a- $\mathbf{e}^{\prime \prime}$ ) Projections from confocal stacks of the CNS at embryonic stage 16, ventral view. Apoptotic cells in red (CM1), whereas glia (in green) are labeled with different markers. Bar $20 \mu \mathrm{m}$. In control repoGal4 embryo (a-a") apoptotic particles are mostly inside anti-SIMU-labeled glia. In the simu mutant $\left(\mathbf{b}-\mathbf{b}^{\prime \prime}\right)$, many apoptotic particles are outside glia labeled with simu-cytGFP. (d- $\mathbf{d}^{\prime \prime}$ ) In the drpr

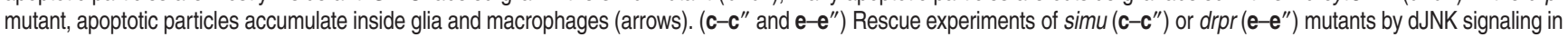
glia (repoGal4:: hepCA). No rescue is detected in the simu mutant ( $\mathbf{c}-\mathbf{c}^{\prime \prime}$ and $\left.\mathbf{f}\right)$ but substantial rescue is detected in the drpr mutant (e- $\mathbf{e}^{\prime \prime}$ and $\left.\mathbf{f}\right)$. Many apoptotic particles accumulate in macrophages outside the CNS (arrows) but not in glia $\left(\mathbf{e}^{\prime \prime}\right)$. (f) Quantification of phenotypic rescue of simu and drpr mutants by repoGal4::hepCA. Columns represent mean total volume of apoptotic particles within confocal stacks of the CNS, \pm S.E.M., $n=5-9$; asterisks indicate statistical significance versus simu and drpr mutants, as determined by the Student's $t$-test, ${ }^{\star *} P<0.002$, NS (not significant) $P>0.05$

damaged or aberrant material during CNS development and injury.

In a normal adult CNS, there is almost no cell death and glial phagocytosis of dying neurons. However, it has been recently shown that after neuronal injury, Drosophila glia upregulate their basal ability to phagocytose through activation of the
dJNK pathway, leading to elevation of DRPR levels. ${ }^{9}$ In contrast, during late embryogenesis, massive neuronal apoptosis takes place, and highly phagocytic glia efficiently clear the apoptotic neurons. At this stage, the phagocytic receptors SIMU and DRPR are highly expressed in glia and have a crucial role in engulfment and degradation of apoptotic 

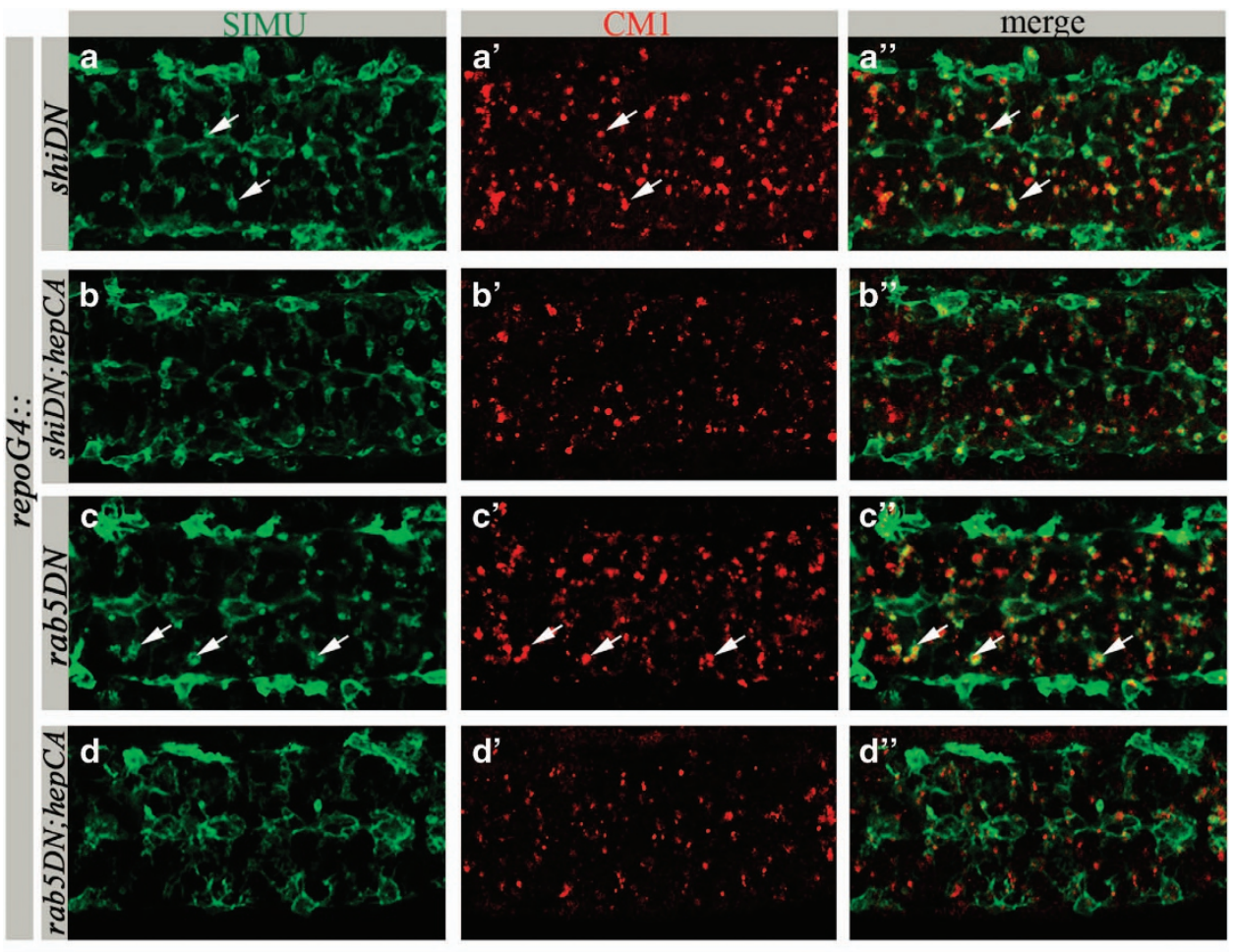

e

Volume of apoptotic particles (\%)
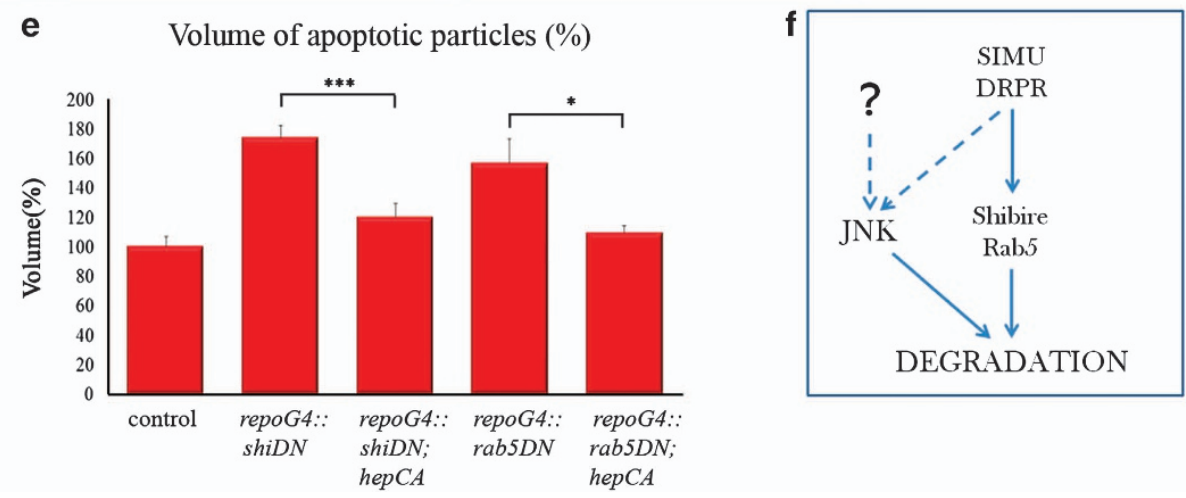

Figure 7 dJNK signaling in glia rescues shibire- and rab5- associated defects in apoptotic particle degradation. (a-d") Projections from confocal stacks of the CNS at embryonic stage 16, ventral view; apoptotic cells are in red (CM1) and glia labeled with anti-SIMU in green. Bar $20 \mu \mathrm{m}$. repoGal4::shibireDN (a-a") and repoGal4::rab5DN (c-c") embryos show an increased volume of apoptotic particles compared with control (Figures $6 a^{\prime}$ and a"), which are found mostly inside SIMU-labeled glia (arrows). In rescue experiments with repoGal4::hepCA (b-b" and $\mathbf{d}-\mathbf{d}^{\prime \prime}$ ) a substantial rescue is detected. (e) Quantification of phenotypic rescue of shibire and rab5 dominant-negative mutants by constitutively active HEP. Columns represent mean total volume of apoptotic particles within confocal stacks of the CNS, \pm S.E.M., $n=6-8$; asterisks indicate statistical significance versus repoGal4:::shibireDN and repoGal4::rab5DN, as determined by the Student's $t$-test, ${ }^{* \star} P<0.002,{ }^{*} P<0.03$. (f) Schematic representation of dJNK signaling in glia downstream of SIMU and DRPR

cells. ${ }^{13,15}$ We have recently demonstrated that embryonic glia are able to clear excess apoptotic particles without increasing SIMU and DRPR levels, and that their expression in the embryonic CNS is controlled by the glial developmental program. ${ }^{15}$ Here we show that this developmental program normally does not involve dJNK pathway activation. Moreover, we demonstrate that ectopic activation of dJNK signaling in embryonic glia does not affect the levels of SIMU and DRPR.

However, we discovered that overexpression of the activated dJNK pathway in embryonic neurons leads to dJNK-mediated upregulation of glial phagocytosis, suggesting that dJNK signaling prepares neighboring glia for removing damaged neuronal material. Importantly, neuronal caspase activation induced by head involution detective (HID) or Reaper did not activate the dJNK pathway in embryonic glia (Supplementary Figure S5), demonstrating that enhanced glial ability to phagocytose is not triggered by neuronal apoptosis per se. A similar mechanism was proposed in elimination of oncogenic cells in Drosophila imaginal epithelia, where dJNK signaling in tumor cells induced their dJNK-dependent phagocytosis by neighboring cells. ${ }^{44}$ In addition, JNK pathway activity has been detected in mammalian macrophages and epithelial cells during engulfment of apoptotic cells in vitro, ${ }^{45,46}$ suggesting a conserved role for JNK signaling in phagocytosis.

Importantly, despite the robust gain-of-function of dJNK signaling in embryonic neurons, only a relatively small number of neurons died and were removed by phagocytic glia. This suggests that activation of the dJNK pathway alone 
is not sufficient to kill developing neurons, and other signals are probably required to cause neural cell death. The additional scenario might be repression of dJNK signaling in neurons by developmental regulators, similar to the Decapentaplegic-mediated protection of epithelial cells from dJNK-induced apoptosis during dorsal closure. ${ }^{47}$

As mentioned above, previous studies demonstrated that dJNK signaling in phagocytic cells upregulates expression of the phagocytic receptor DRPR. ${ }^{9,48}$ Our data demonstrate that dJNK pathway activation in embryonic glia does not elevate the levels of SIMU and DRPR, suggesting that they are not a limiting factor for glial phagocytosis during embryogenesis. However, we demonstrate the ability of dJNK signaling to substantially rescue abnormal degradation of apoptotic particles inside glial cells downstream of DRPR function. These results argue that during late embryogenesis, dJNK signaling is able to upregulate glial phagocytosis through acceleration of the intracellular degradation machinery, and demonstrate for the first time the involvement of dJNK signaling in degradation of apoptotic particles.

The physiological or pathological conditions under which JNK signaling connects neuronal damage and upregulation of glial phagocytosis are currently unclear, and require additional studies. However, it is likely that when the trigger for dJNK signaling overactivation in embryonic neurons is identified it will work through a mechanism consistent with the data provided here. Various types of intrinsic and environmental stresses activate the JNK pathway in mammalian neurons, leading to neuronal death, such as UV irradiation, reactive oxygen species, DNA damage, heat shock, bacterial antigens, and inflammatory cytokines. ${ }^{49}$ In Drosophila, JNK signaling has been shown to have a critical role in elimination of aberrant cells during development. ${ }^{18}$ Moreover, in the Drosophila eye model of Alzheimer disease, JNK signaling was induced in neurons by accumulation of $A \beta 42$ plaques, which contributed to $A \beta 42-$ mediated cell death. ${ }^{50}$ It would be intriguing to evaluate the state of glia in similar situations of neuronal death. More competent phagocytic glia could be an advantage under neuronal stress conditions in the developing and adult CNS.

\begin{abstract}
Materials and Methods
Fly strains. The following fly strains were used in this work: repoGal4 (B Jones), elavGal4 (O Schuldiner), UAScytGFP (\#1521; Bloomington), UASnGFP (\#4775; Bloomington), TRE-eGFP (D Bohmann), UAShid and UASreaper (E Arama), simu and simu-cytGFP, ${ }^{13} \mathrm{drpr}$ (M Freeman), tubGal80 ${ }^{\text {ts }}$ (\#7019; Bloomington), UASrab5S43N (\#42703; Bloomington), UASshiK44A (\#5811; Bloomington), puclacZ $=$ puc[E69] (A Salzberg). P\{A92\}puEE69 mutant embryos comprise a lacZ-containing P-element inserted into the puc endogenous locus, which disrupts puc normal function. ${ }^{51}$ UAShepCA (\#9306, \#6406; Bloomington), UASbskK53R= bskDN (\#9311; Bloomington). For neuron-specific expression of elavGal4, embryos were placed at $18^{\circ} \mathrm{C}$ until early stage 14 and was then transferred to $29^{\circ} \mathrm{C}$ for $4 \mathrm{~h}$. For hid expression in larval neurons, we placed elavGa4::hid; tubGal $80^{\text {ts }}$ progeny at $18^{\circ} \mathrm{C}$ until the 2nd instar larval stage and then shifted them to $29^{\circ} \mathrm{C}$ for $24 \mathrm{~h}$.
\end{abstract}

Immunohistochemistry and live imaging. Guinea pig anti-DRPR antibody was raised against a GST-tagged fragment comprising the last 220 amino acids of the DRPR protein. For immunohistochemistry, embryos were fixed and stained according to standard procedures. Guinea pig anti-SIMU ${ }^{25}$ and guinea pig anti-DRPR were used at a 1:5000 and 1:100 concentrations, respectively. Rabbit anti-activated caspase 3 (CM1, BD, Franklin Lakes, NJ, USA) and mouse anti-GFP (Roche, Mannheim, Germany) were used at 1:50 and 1:100 concentrations, respectively. Rabbit anti-dJUN was a gift from $D$ Bohmann and used at 1:400 dilution. Mouse anti-REPO (1:20), anti-CUT (1:50), anti-EVE $(1: 100)$ and anti-DAC $(1: 5)$ were from Hybridoma bank (lowa city, IA, USA). Fluorescent secondary antibodies Cy3/488/647 from Jackson ImmunoResearch (West Grove, PA, USA) were used at 1: 200 dilutions. Glycerol solution (80\%) was used as the imaging medium. All confocal images were acquired on a confocal microscope Zeiss (Aalen, Germany) LSM 700 using a Plan-Apochromat $\times 20 / 0.8$ M27 lens. Image analysis was performed using Zeiss LSM 700 and Imaris (Bitplane, Zurich, Switzerland) software. To quantitate the volume of apoptotic particles or the number of glial cells, confocal stacks (five sections; total $7.5 \mu \mathrm{m}$ ) were acquired from the neural cortex of stage-16 ventral nerve cords. To count DAC- or EVEpositive neurons, confocal stacks (15 sections; total $22.5 \mu \mathrm{m}$ ) of three abdominal segments were acquired. To count CUT-positive neurons, confocal stacks (four sections; total $6 \mu \mathrm{m}$ ) of three abdominal segments were acquired.

Live imaging was carried out following dechorionation of stage-15 embryos. LysoTracker (Molecular Probes, Leiden, The Netherlands) was injected at 2-3\% of the egg volume and mounted in Halocarbon oil, as described. ${ }^{52}$

\section{Conflict of Interest}

The authors declare no conflict of interest.

Acknowledgements. We would like to thank B Jones, O Schuldiner, D Bohmann, E Arama, M Freeman, A Salzberg, Hybridoma bank and the Bloomington Stock Center for generously providing fly strains and antibodies. We thank A Salzberg and T Schultheiss for comments on the manuscript, and the Kurant laboratory members for constructive criticism and support. We also thank E Suss-Toby at the Interdepartmental Bioimaging facility for excellent technical support. We gratefully acknowledge financial support from the Israel Science Foundation (grant no 427/11) and from Allen and Jewell Prince Center for Neurodegenerative Disorders of the Brain.

1. Raff MC, Barres BA, Burne JF, Coles HS, Ishizaki Y, Jacobson MD. Programmed cell death and the control of cell survival: lessons from the nervous system. Science 1993; 262: 695-700.

2. Marin-Teva JL, Cuadros MA, Martin-Oliva D, Navascues J. Microglia and neuronal cell death. Neuron Glia Biol 2011; 7: 25-40.

3. Buss RR, Oppenheim RW. Role of programmed cell death in normal neuronal development and function. Anat Sci Int 2004; 79: 191-197.

4. Buss RR, Sun W, Oppenheim RW. Adaptive roles of programmed cell death during nervous system development. Annu Rev Neurosci 2006; 29: 1-35.

5. Schlegelmilch T, Henke K, Peri F. Microglia in the developing brain: from immunity to behaviour. Curr Opin Neurobiol 2011; 21: 5-10.

6. Amor S, Puentes F, Baker D, van der Valk P. Inflammation in neurodegenerative diseases. Immunology 2010; 129: 154-169.

7. Farina $\mathrm{C}$, Aloisi $\mathrm{F}$, Meinl E. Astrocytes are active players in cerebral innate immunity. Trends Immunol 2007; 28: 138-145.

8. MacDonald JM, Beach MG, Porpiglia E, Sheehan AE, Watts RJ, Freeman MR. The Drosophila cell corpse engulfment receptor Draper mediates glial clearance of severed axons. Neuron 2006; 50: 869-881.

9. Macdonald JM, Doherty J, Hackett R, Freeman MR. The c-Jun kinase signaling cascade promotes glial engulfment activity through activation of draper and phagocytic function. Cell Death Differ 2013; 20: 1140-1148.

10. Hartenstein V. Morphological diversity and development of glia in Drosophila. Glia 2011; 59 : 1237-1252.

11. Parker RJ, Auld VJ. Roles of glia in the Drosophila nervous system. Semin Cell Dev Biol 2006; 17: 66-77.

12. Freeman MR, Doherty J. Glial cell biology in Drosophila and vertebrates. Trends Neurosci 2006; 29: 82-90.

13. Kurant E, Axelrod S, Leaman D, Gaul U. Six-microns-under acts upstream of Draper in the glial phagocytosis of apoptotic neurons. Cell 2008; 133: 498-509.

14. Freeman MR, Delrow J, Kim J, Johnson E, Doe CQ. Unwrapping glial biology: Gcm target genes regulating glial development, diversification, and function. Neuron 2003; 38: 567-580.

15. Shklyar B, Sellman Y, Shklover J, Mishnaevski K, Levy-Adam F, Kurant E. Developmental regulation of glial cell phagocytic function during Drosophila embryogenesis. Dev Biol 2014; 393: 255-269.

16. Riesgo-Escovar JR, Jenni M, Fritz A, Hafen E. The Drosophila Jun-N-terminal kinase is required for cell morphogenesis but not for DJun-dependent cell fate specification in the eye. Genes Dev 1996; 10: 2759-2768.

17. Sluss HK, Han Z, Barrett T, Goberdhan DC, Wilson C, Davis RJ et al. A JNK signal transduction pathway that mediates morphogenesis and an immune response in Drosophila. Genes Dev 1996; 10: 2745-2758. 
18. Igaki T. Correcting developmental errors by apoptosis: lessons from Drosophila JNK signaling. Apoptosis 2009; 14: 1021-1028.

19. Kuan CY, Yang DD, Samanta Roy DR, Davis RJ, Rakic P, Flavell RA. The Jnk1 and Jnk2 protein kinases are required for regional specific apoptosis during early brain development Neuron 1999; 22: 667-676.

20. Lee JH, Koh H, Kim M, Park J, Lee SY, Lee S et al. JNK pathway mediates apoptotic cell death induced by tumor suppressor LKB1 in Drosophila. Cell Death Differ 2006; 13: 1110-1122.

21. Rogulja-Ortmann A, Lüer K, Seibert J, Rickert C, Technau GM. Programmed cell death in the embryonic central nervous system of Drosophila melanogaster. Development 2007; 134: $105-116$

22. Moreno E, Yan M, Basler K. Evolution of TNF signaling mechanisms: JNK-dependent apoptosis triggered by Eiger, the Drosophila homolog of the TNF superfamily. Curr Bio 2002; 12: 1263-1268.

23. Igaki T, Kanda H, Yamamoto-Goto Y, Kanuka H, Kuranaga E, Aigaki T et al. Eiger, a TNF superfamily ligand that triggers the Drosophila JNK pathway. Embo J 2002; 21: 3009-3018.

24. Martin-Blanco E, Gampel A, Ring J, Virdee K, Kirov N, Tolkovsky AM et al. puckered encodes a phosphatase that mediates a feedback loop regulating JNK activity during dorsal closure in Drosophila. Genes Dev 1998; 12: 557-570.

25. Shklyar B, Levy-Adam F, Mishnaevski K, Kurant E. Caspase activity is required for engulfment of apoptotic cells. Mol Cell Biol 2013; 33: 3191-3201.

26. Chatterjee N, Bohmann D. A versatile PhiC31 based reporter system for measuring AP-1 and Nrf2 signaling in Drosophila and in tissue culture. PLoS One 2012; 7: e34063.

27. Berger C, Renner S, Luer K, Technau GM. The commonly used marker ELAV is transiently expressed in neuroblasts and glial cells in the Drosophila embryonic CNS. Dev Dyn 2007 236: 3562-3568.

28. Alvarez-Dominguez C, Barbieri AM, Beron W, Wandinger-Ness A, Stahl PD. Phagocytosed live Listeria monocytogenes influences Rab5-regulated in vitro phagosome-endosome fusion. J Biol Chem 1996; 271: 13834-13843.

29. Duclos S, Diez R, Garin J, Papadopoulou B, Descoteaux A, Stenmark H et al. Rab5 regulates the kiss and run fusion between phagosomes and endosomes and the acquisition of phagosome leishmanicidal properties in RAW 264.7 macrophages. J Cell Sci 2000; 113 3531-3541.

30. Claus V, Jahraus A, Tjelle T, Berg T, Kirschke H, Faulstich $\mathrm{H}$ et al. Lysosomal enzyme trafficking between phagosomes, endosomes, and lysosomes in $\mathrm{J} 774$ macrophages. Enrichment of cathepsin H in early endosomes. J Biol Chem 1998; 273: 9842-9851.

31. Yu X, Odera S, Chuang CH, Lu N, Zhou Z. C. elegans Dynamin mediates the signaling of phagocytic receptor CED-1 for the engulfment and degradation of apoptotic cells. Dev Cell 2006; 10: 743-757.

32. Kinchen JM, Doukoumetzidis $\mathrm{K}$, Almendinger J, Stergiou L, Tosello-Trampont A Sifri $C D$ et al. A pathway for phagosome maturation during engulfment of apoptotic cells. Nat Cell Biol 2008; 10: 556-566.

33. Yu X, Lu N, Zhou Z. Phagocytic receptor CED-1 initiates a signaling pathway for degrading engulfed apoptotic cells. PLOS Biol 2008; 6: e61.

34. Kitano M, Nakaya M, Nakamura T, Nagata S, Matsuda M. Imaging of Rab5 activity identifies essential regulators for phagosome maturation. Nature 2008; 453: 241-245.

35. Hakim Y, Yaniv SP, Schuldiner O. Astrocytes play a key role in Drosophila mushroom body axon pruning. PLoS One 2014; 9: e86178.

36. Awasaki T, Ito K. Engulfing action of glial cells is required for programmed axon pruning during Drosophila metamorphosis. Curr Biol 2004; 14: 668-677.
37. Broadie K. Axon pruning: an active role for glial cells. Curr Biol 2004; 14: R302-R304.

38. Tasdemir-Yilmaz OE, Freeman MR. Astrocytes engage unique molecular programs to engulf pruned neuronal debris from distinct subsets of neurons. Genes Dev 2013; 28: 20-33.

39. Watts RJ, Schuldiner O, Perrino J, Larsen C, Luo L. Glia engulf degenerating axons during developmental axon pruning. Curr Biol 2004; 14: 678-684.

40. Stronach BE, Perrimon N. Stress signaling in Drosophila. Oncogene 1999; 18: 6172-6182.

41. Chen F. JNK-induced apoptosis, compensatory growth, and cancer stem cells. Cancer Res 2012; 72: 379-386

42. Leppa S, Bohmann D. Diverse functions of JNK signaling and c-Jun in stress response and apoptosis. Oncogene 1999; 18: 6158-6162.

43. Dhanasekaran DN, Reddy EP. JNK signaling in apoptosis. Oncogene 2008; 27: 6245-6251.

44. Ohsawa S, Sugimura K, Takino K, Xu T, Miyawaki A, Igaki T. Elimination of oncogenic neighbors by JNK-mediated engulfment in Drosophila. Dev Cell 2011; 20: 315-328.

45. Patel VA, Lee DJ, Feng L, Antoni A, Lieberthal W, Schwartz JH et al. Recognition of apoptotic cells by epithelial cells: conserved versus tissue-specific signaling responses. J Biol Chem 2010; 285: 1829-1840.

46. Patel VA, Longacre A, Hsiao K, Fan H, Meng F, Mitchell JE et al. Apoptotic cells, at all stages of the death process, trigger characteristic signaling events that are divergent from and dominant over those triggered by necrotic cells: Implications for the delayed clearance model of autoimmunity. J Biol Chem 2006; 281: 4663-4670.

47. Beira JV, Springhorn A, Gunther S, Hufnagel L, Pyrowolakis G, Vincent JP. The Dpp/ TGFbeta-dependent corepressor Schnurri protects epithelial cells from JNK-induced apoptosis in Drosophila embryos. Dev Cell 2014; 31: 240-247.

48. Etchegaray Jl, Timmons AK, Klein AP, Pritchett TL, Welch E, Meehan TL et al. Draper acts through the JNK pathway to control synchronous engulfment of dying germline cells by follicular epithelial cells. Development 2012; 139: 4029-4039.

49. Harper SJ, LoGrasso P. Signalling for survival and death in neurones: the role of stress-activated kinases, JNK and p38. Cell Signal 2001; 13: 299-310.

50. Tare M, Modi RM, Nainaparampil JJ, Puli OR, Bedi S, Fernandez-Funez P et al. Activation of JNK signaling mediates amyloid-ss-dependent cell death. PLoS One 2011; 6: e24361.

51. Ring JM, Martinez Arias A. puckered, a gene involved in position-specific cell differentiation in the dorsal epidermis of the Drosophila larva. Dev Suppl 1993: 251-259.

52. Shklyar B, Shklover J, Kurant E. Live imaging of apoptotic cell clearance during Drosophila embryogenesis. J Vis Exp 2013; 18: 78

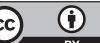

Cell Death and Disease is an open-access journal published by Nature Publishing Group. This work is licensed under a Creative Commons Attribution 4.0 International Licence. The images or other third party material in this article are included in the article's Creative Commons licence, unless indicated otherwise in the credit line; if the material is not included under the Creative Commons licence, users will need to obtain permission from the licence holder to reproduce the material. To view a copy of this licence, visit http://creativecommons.org/licenses/by/4.0 